\title{
Mental Health Difficulties, Attainment \& Attendance: A Cross-sectional Study
}

Suzet Tanya Lereya, PhD, Evidence-Based Practice Unit, University College London and Anna Freud National Centre for Children and Families, UK;

Meera Patel, BSc, Child Outcomes Research Consortium, Anna Freud National Centre for Children and Families, UK;

Joao Pedro Garcez Aurelio dos Santos, PhD, Manchester Institute of Education, University of Manchester, UK;

Jessica Deighton, PhD, Evidence-Based Practice Unit, University College London and Anna Freud National Centre for Children and Families, UK.

Acknowledgements: We are extremely grateful to all the students who took part in this study, the local authorities and schools for their help in recruiting them. The authors would like to thank members of CPRU: Terence Stephenson, Catherine Law, Amanda Edwards, Ruth Gilbert, Steve Morris, Helen Roberts, Cathy Street, and Russell Viner.

Funding: The data used in this study was collected as part of the HeadStart learning programme and supported by funding from the Big Lottery Fund. The content is solely the responsibility of the authors and it does not necessarily reflect the views of the Big Lottery Fund. The Policy Research Unit in the Health of Children, Young People and Families is funded by the NIHR Policy Research Programme. This paper is based on independent research commissioned and funded by the National Institute for Health Research Policy Research Programme. The views expressed are those of the author(s) and not necessarily those of the NHS, the National Institute for Health Research, the Department of Health and Social Care or its arm's length bodies, and other Government Departments. Jessica Deighton was supported by the National Institute for Health Research (NIHR) Collaboration for 
Leadership in Applied Health Research and Care (CLAHRC) North Thames at Bart's Health NHS Trust.

Correspondence: Dr Suzet Tanya Lereya, Evidence-Based Practice Unit, University College London and Anna Freud National Centre for Children and Families, 47 Brunswick Place, London, N1 6EB, Phone: 0207433 6193, Email: Tanya.lereya@annafreud.org 


\begin{abstract}
Evidence for the association between mental health difficulties and academic outcomes is sparse and shows mixed results. The aim of this study was to investigate the association between educational attainment, absenteeism and mental health difficulties while controlling for various child characteristics such as special education needs and socio-economic background. 15,301 year 7 pupils (mean age: 11.91; $\mathrm{SD}=0.28$ ) from England completed the Strengths and Difficulties Questionnaire. Attainment, persistent absenteeism and child characteristics were derived from the national pupil database. Multilevel regression analysis showed that mental health difficulties were negatively associated with attainment and positively associated with persistent absenteeism. When all mental health difficulties were modelled simultaneously, behavioural difficulties, hyperactivity/attention difficulties and difficulties with peers were negatively associated with attainment. Emotional difficulties and hyperactivity/attention difficulties were positively associated with persistent absenteeism. The results of the current study highlight the importance of integration between mental health support and policy creation in relation to mental health difficulties and wellbeing in schools.
\end{abstract}

Key words: Mental health difficulties; attainment; absenteeism 


\section{Mental Health Difficulties, Attainment \& Attendance: A Cross-sectional Study}

Mental health difficulties affect $10-20 \%$ of children and adolescents worldwide and have implications for various long-term outcomes such as health, education, employment and wellbeing [1]. Similarly, educational outcomes (such as attainment) in childhood are associated with many later-life social and economic outcomes, including occupation, earnings and health [2].

Previous research has shown a consistent negative association between externalising problems and educational attainment but evidence for the associations between internalising problems and educational attainment has produced mixed results [3]. Only a few studies focused on school absenteeism and mental health predominantly included multiple risk factors and controlled for other associated factors [4,5]. Moreover, the majority of the research was conducted in the US, making it harder to interpret the results in Europe due to the differences in welfare systems [3].

There is an increasing focus on schools as a site for prevention and early intervention in mental health problems [6]. However, schools mainly target academic outcomes with performance metrics focused specifically on academic attainment and attendance records. It is important to investigate the relationships between mental health and academic outcomes to highlight the potential benefits of improving mental health on these academic metrics. Thus, the aim of this study was to investigate the association between educational attainment, absenteeism and mental health difficulties while controlling for various child level characteristics. 
Methods

Sample

Data were collected in 2017 from children involved in the project HeadStart: a five-year, $£ 56$ million National Lottery funded programme set-up by the Big Lottery Fund, aiming to explore and test new ways to improve the mental health and wellbeing of young people aged 10 to 16 and prevent serious mental health issues from developing [7]. Six local authority led HeadStart partnerships in Blackpool, Cornwall, Hull, Kent, Newham and Wolverhampton are working with local young people, schools, families, charities, community and public services to deliver their local programme. Schools have been selected by these partnerships; in most cases driven by perceived need in these schools but also by the potential for schools to successfully engage with the programme. Pupils in participating schools complete an annual survey (the Wellbeing Measurement Framework) and the results presented are based on the baseline data. The analyses reported are based on 15,301 (of 15,860) year 7 pupils $(46.5 \%$ male; mean age $=11.91, \mathrm{SD}=0.28)$ from 97 mainstream schools (including state, academy and free schools) who completed the survey and had National Pupil Data. The sample was not drawn to be representative; it was based on local areas that were part of the programme. Overall, 11,768 (78.2\%) of pupils were White (national state funded secondary schools' average $75.1 \%$ ); $17.3 \%$ were eligible for free school meals (FSM) (national state funded secondary schools' average 13.8\%); $12.6 \%$ had a special educational need with or without statement (not including Education, Health and Care (EHC) plans) (national state funded secondary schools' average 12.4\%, including EHC plans). The special educational need follows a graduated approach to helping children in need of special provision. The first stage is providing SEN support (without statement) and the second stage involves the Local Authority, with a statement granted for the most serious cases (with a statement). All statements are currently being changed to EHC plans. 5.8\% had child in need status, referring 
to children's social care, most frequently because of concerns about abuse or neglect, acute family stress or familial dysfunction.

\section{Measures}

Emotional, behavioural, and hyperactivity/attention difficulties and difficulties with peers were measured with self-reported Strengths and Difficulties Questionnaire [8]. Total difficulties score was calculated by summing all the sub-scales. Those scoring above the borderline threshold on a subscale were considered as having high levels of the respective difficulty. In the current study, internal consistency of the subscales ranged from $\alpha=0.60$ 0.74 , total difficulties $\alpha=0.81$.

Attainment and persistent absenteeism were derived from the national pupil database (NPD). Attainment was measured with the Key Stage 2 standardised national tests (taken by 11-yearolds in the final year of primary school) for English (reading and writing) and mathematics. 80 is the lowest score that can be awarded and 120 is the highest score. Pupils scoring at least 100 will have met the expected standard. Consistent with UK government's definition, persistent absenteeism was considered present if a pupil missed $10 \%$ or more of their possible sessions during the 2015/16 academic year.

Special educational needs (SEN), ever being eligible for FSM, ethnicity and CIN status were derived from the NPD. Children with SEN, with or without statement, were considered as having SEN. FSM is frequently used as an indicator of low family income since only families on income support are eligible.

Procedure 
Ethical approval was obtained from the University College London Research Ethics Committee (UCL Ethics 8097/003). Questionnaires were completed using a secure online system during a usual school day with parent consent and child assent.

\section{Analyses}

To identify the association between mental health difficulties and attainment, five multilevel linear regression models were used (Table 1) and to identify the association between mental health difficulties and persistent absenteeism, five multilevel logistic regression analyses were run (Table 2). Single regression analyses (separate for attainment and absenteeism) were run where all mental health difficulties were entered into the models simultaneously (Tables $1 \& 2$, model VI). Random effects linear/logistic regression analyses (allowing for different school intercepts) were run using STATA version14; unstandardised betas, and standard errors are reported for attainment models and odds ratios with $95 \%$ confidence intervals are reported for absenteeism models. All analyses controlled for sex, age, ethnicity, SEN, FSM and CIN.

\section{Results}

Overall, 3,833 (25.7\%) were above the borderline threshold on emotional difficulties; 4,338 $(29.0 \%)$ were above the threshold on behavioural difficulties; 5,421 (36.3\%) were above the threshold on hyperactivity/attention difficulties; 3,780 (25.3\%) were above the threshold on peer difficulties. 5,273 (35.4\%) were above the threshold on total difficulties score. The mean Key Stage 2 national test score was $103.12(\mathrm{SD}=6.45)$ and $961(6.40 \%)$ had an overall absence rate of $10 \%$ or higher. There was a significant difference in the Key Stage 2 national test scores for those who were not persistently absent $(M=103.3, S D=6.40)$ and those who were persistently absent $(\mathrm{M}=100.3, \mathrm{SD}=6.49) ; \mathrm{t}(14,449)=13.42, \mathrm{p}<.0001$. 
All mental health difficulties were negatively associated with attainment and positively associated with persistent absenteeism. When all mental health difficulties were entered into the model simultaneously, behavioural difficulties, hyperactivity/attention difficulties and difficulties with peers were negatively associated with attainment. Emotional difficulties and hyperactivity/attention difficulties were positively associated with persistent absenteeism.

From the covariates, being Asian (compared to being White) and being older were related with higher attainment; on the other hand, having SEN, FSM eligibility and having CIN status were associated with lower attainment across all models. Being older, having SEN, FSM eligibility and having CIN status increased the odds of being persistently absent whereas being Asian (compared to being White) and being Black (compared to being White) decreased these odds.

\section{[INSERT TABLES $1 \& 2]$}

\section{Discussion}

The current study aimed to investigate the association between mental health difficulties and educational outcomes while controlling for various child characteristics. Results showed mental health difficulties were associated with both academic attainment and persistent absenteeism. Specific mental health problems differentially predicted these two outcomes such that externalising problems (behavioural difficulties and hyperactivity/attention) and peer problems were associated with attainment and emotional difficulties, and hyperactivity/attention difficulties were associated with attendance once all mental health difficulties were added into the model simultaneously.

Findings are consistent with previous literature showing that attainment scores of persistent absentees were lower than attenders. This might be due to absent pupils receiving fewer 
hours of instructions or feeling a greater sense of alienation from peers and teachers on their return, which may impact on learning [9]. Moreover, persistent absentees were more likely to be older [5]. This might be due to older children being more capable of finding ways to avoid going to school. On the other hand, older children were more likely to have better attainment scores which is in line with literature suggesting that age-position of a pupil within a class has a major impact on their performance [10]. The investigation between mental health difficulties and attainment and attendance suggested that children who show externalising difficulties have a greater risk of 'acting out' and/or being dismissed from class and receiving negative feedback, which might increase the risk of lower educational attainment [11]. In support of the association between peer problems and attainment, children's social experiences at school have previously been shown to affect their academic performance [12]. The fact that emotional difficulties were no longer associated with academic attainment when all mental health difficulties were added into the model simultaneously might be due to the co-occurrence of internalising difficulties with externalising and hyperactivity/attention difficulties [13]. Regarding attendance, similar to previous literature [14], emotional and hyperactivity/attention difficulties were associated with persistent absenteeism. This relationship remained significant even when all mental health difficulties were added into the model which might be due to those with internalising problems being more likely to display social anxiety, depression or somatic complains which in return may result in more absences [14].

It is important to note the methodological limitations of the study. Firstly, the study sample was not representative of all school children in England. Secondly, due to the cross-sectional nature of this study, we cannot disentangle temporal precedence between educational outcomes and mental health difficulties. Future studies should apply longitudinal methods. 
Notwithstanding these limitations, this study highlighted the negative association between mental health difficulties and educational outcomes, supporting the importance of policy and practice initiatives to support better links between schools and mental health services and mental health providers to consider educational outcomes as potential indicators of mental health difficulties. 
Declaration of Conflicting Interests: On behalf of all authors, the corresponding author states that there is no conflict of interest. 


\section{References}

1. Kieling C, Baker-Henningham H, Belfer M, Conti G, Ertem I, Omigbodun O, Rohde LA, Srinath S, Ulkuer N, Rahman A (2011) Child and adolescent mental health worldwide: Evidence for action. Lancet 378 (9801):1515-1525. doi:http://dx.doi.org/10.1016/S0140-6736(11)60827-1

2. Crystal S, Shea D, Krishnaswami S (1992) Educational attainment, occupational history, and stratification: determinants of later-life economic outcomes. J Gerontol 47:213-221

3. Veldman K, Bültmann U, Stewart RE, Ormel J, Verhulst FC, Reijneveld SA (2014) Mental health problems and educational attainment in adolescence: 9-year follow-up of the TRAILS study. PLoS ONE 9 (7):e101751. doi:10.1371/journal.pone.0101751

4. Ingul JM, Klöckner CA, Silverman WK, Nordahl HM (2012) Adolescent school absenteeism:

Modelling social and individual risk factors. 17 (2):93-100. doi:10.1111/j.1475-3588.2011.00615.x 5. Pflug V, Schneider S (2016) School absenteeism: An online survey via social networks. 47 (3):417429. doi:10.1007/s10578-015-0576-5

6. Weare K, Nind M (2011) Mental health promotion and problem prevention in schools: What does the evidence say? 26:i29-i69

7. Big Lottery Fund (2014) Fulfilling Lives: HeadStart. https://www.biglotteryfund.org.uk/globalcontent/programmes/england/fulfilling-lives-headstart. Accessed 15 February 2018

8. Goodman R, Meltzer H, Bailey V (1998) The strengths and difficulties questionnaire: A pilot study on the validity of the self-report version. Eur Child Adolesc Psychiatry 7 (3):125-130.

doi:10.1007/s007870050057

9. Gottfried MA (2011) The detrimental effects of missing school: Evidence from urban siblings. Am J Educ 117 (2):147-182. doi:10.1086/657886

10. Bell JF, Daniels S (1990) Are summer-born children born disadvantaged? The birthdate effect in education, . Oxford Review of Education 16:67-80

11. Henry KL, Huizinga DH (2007) School-related risk and protective factors associated with truancy among urban youth placed at risk. J Prim Prev 28 (6):505-519. doi:10.1007/s10935-007-0115-7

12. Buhs ES, Ladd GW, Herald SL (2006) Peer exclusion and victimization: Processes that mediate the relation between peer group rejection and children's classroom engagement and achievement? J Educ Psychol 98 (1):1-13. doi:10.1037/0022-0663.98.1.1

13. Breslau J, Lane M, Sampson N, Kessler RC Mental disorders and subsequent educational attainment in a US national sample. J Psychiatr Res 42 (9):708-716.

doi:10.1016/j.jpsychires.2008.01.016

14. Kearney CA (2008) School absenteeism and school refusal behavior in youth: A contemporary review. Clin Psychol Rev 28 (3):451-471. doi:https://doi.org/10.1016/i.cpr.2007.07.012 